\title{
Quelques réflexions sur les possibilités de l'enseignement de I'histoire des mathématiques dans la formation des enseignants en Hongrie
}

\author{
Katalin Gosztonyi ${ }^{\mathrm{a}}$ \\ Bolyai Intézet, Université de Szeged, Hongrie ; Laboratoire de didactique André Revuz, Université \\ Paris Diderot, France
}

\begin{abstract}
Résumé. Je présente dans cet article des réflexions concernant un projet d'enseignement motivé d'une part par une demande institutionnelle, d'autre part par mes expériences et recherches personnelles. Je vais aborder (1) la question des objectifs possibles d'un cours d'histoire des mathématiques dans la formation des enseignants ; (2) les contraintes propres au contexte hongrois, tout particulièrement concernant les sources sur lesquelles un tel cours peut être basé ; enfin (3) les idées émergeant de mes recherches actuelles sur l'histoire d'une tradition hongroise de l'enseignement des mathématiques, susceptibles de répondre à une grande partie des objectifs et contraintes présentés. J'utilise le mode narratif pour rendre compte de la dynamique des interactions entre expériences d'enseignement, rencontre avec des expériences et recherches internationales, et recherches personnelles : une dynamique qui peut permettre de concevoir progressivement un enseignement adapté à son contexte particulier.
\end{abstract}

\begin{abstract}
I present in this article some reflections concerning a course project whose realization is motivated by an institutional demand and also by my personal experiences and research. I first deal with the questions of the possible objectives of a history of mathematics course in the teacher training. I then examine the difficulties created by the particular conditions of Hungary with special regard to the sources of such a course. I finally explain some ideas emerging from my actual researches about the history of a Hungarian tradition of mathematics education, which may be suitable to answer to most of the objectives and difficulties presented. I will follow a narrative order in the presentation, to show a dynamic between teaching experiences, the encounter with international experiences and researches, and personal researches. Such a dynamic may help to progressively design a course fitting to its particular context.
\end{abstract}

Les réflexions présentées dans cet article sont nées de deux différents types de motivation : d'ordre institutionnel et d'ordre personnel. Le motif institutionnel est lié à l'introduction obligatoire d'un nouveau cours d'histoire des mathématiques pour la formation des enseignants de mathématiques,

\footnotetext{
a e-mail : katalin.gosztonyi@gmail.com
}

This is an Open Access article distributed under the terms of the Creative Commons Attribution License 4.0, which permits unrestricted use, distribution, and reproduction in any medium, provided the original work is properly cited. 
prescrit il y a quelques années, dans le cadre d'une réforme de la formation des enseignants en Hongrie ${ }^{1}$. En même temps, la construction d'un tel cours pose beaucoup de difficultés, car en Hongrie, les recherches en histoire des mathématiques ont très peu de tradition, et par conséquent, il manque de formateurs compétents dans ce domaine ainsi que des sources primaires et secondaires sur lesquels un tel cours pourrait être basé.

Les réflexions suivantes sont par ailleurs motivées par la curiosité et la carrière personnelle de l'auteur. D'une part, étant doctorante en didactique des mathématiques, je vais, dans le futur, très probablement participer à la formation des enseignants. D'autre part, mes divers essais et expériences pour enseigner l'histoire des mathématiques aux différents niveaux de la scolarité, ainsi que mes recherches actuelles largement ancrées dans une approche historique, me motivent à intégrer cette approche dans ma future activité de formatrice.

Dans la suite du texte, je présente mon parcours personnel, en partant d'une première expérience d'enseignement de l'histoire des mathématiques que j'ai réalisée en tant qu'étudiante, sans connaître d'autres expériences ou recherches sur le sujet. Ce type d'expérience naïve n'est probablement pas rare parmi les enseignants, particulièrement dans le domaine de l'histoire des sciences. Dans une deuxième partie, sans essayer d'offrir un panorama complet ni représentatif des recherches et expériences concernant l'intégration de l'histoire des mathématiques dans l'enseignement, je vais montrer comment la rencontre avec quelques expériences internationales sur le sujet a contribué à développer mes réflexions pour la conception d'un cours de formation des enseignants. Je soulignerai tout particulièrement deux questions : celle des objectifs d'un tel cours et celle de ses sources. Je me référerai en particulier à l'étude ICMI intitulée History in Mathematics Education qui a tenté d'offrir un panorama mondial de l'intégration d'un aspect historique dans l'enseignement des mathématiques, et qui a également consacré un chapitre à la formation des enseignants. ${ }^{2}$

Dans une troisième partie, je présente brièvement mes recherches actuelles, menées dans le cadre d'un doctorat en histoire et en didactique des mathématiques, en montrant comment elles ont émergé de mes premières expériences d'enseignement de l'histoire des mathématiques. En outre je montrerai dans la quatrième partie comment ces recherches enrichissent mes réflexions actuelles sur le projet de cours dont il a été question plus haut. Je proposerai donc une manière de concevoir ce cours qui pourrait d'une part partiellement répondre à des problèmes et questions détaillés dans la seconde partie, et qui est d'autre part inspirée par les recherches présentées dans la troisième partie. Cette conception mériterait sans doute d'être encore précisée et complétée ; toutefois, quelques expériences ponctuelles récentes avec des enseignants français et britanniques semblent en confirmer l'intérêt. L'article se conclut par une présentation de ces expériences.

\section{Les premières expériences personnelles}

Ma première expérience d'enseignement de l'histoire des mathématiques remonte à 2007. Encore étudiante, pour devenir professeur de mathématiques et de philosophie, j'ignorais tout des recherches internationales sur le sujet. Il s'agissait d'un atelier d'histoire des mathématiques pour un groupe de lycéens dans une colonie de vacances. J'avais moi-même élaboré le programme de cet atelier d'une semaine et constitué de onze séances de deux heures chacune. Mes objectifs principaux étaient de travailler sur la conception des mathématiques des élèves, en présentant les mathématiques comme

\footnotetext{
${ }^{1}$ La formation des enseignants a subi plusieurs réformes en Hongrie au cours de ces dernières années. Selon la règle actuelle, les enseignants du secondaire obtiennent leur diplôme, équivalent à un niveau master, après 4 (pour le collège) ou 5 ans (pour le lycée) d'études et une année de stage. Ils enseignent deux matières. La formation comprend des cours dans ces deux matières, des cours de psychologie/pédagogie et des cours de didactique, spécifiques aux matières enseignées. Le cours d'histoire des mathématiques est intégré dans cette dernière partie de didactique des mathématiques.

2 Fauvel, van Maanen, 2000.
} 
un produit de l'activité humaine, en plein développement ; de proposer une approche multiculturelle et interdisciplinaire ; d'évoquer certains sujets qui ne font pas partie du programme ordinaire du lycée, tout en revenant sur des sujets déjà connus afin d'amener les élèves à une compréhension plus profonde à l'aide de l'histoire.

Le programme de l'atelier suivait un ordre plus ou moins chronologique de l'histoire des mathématiques ; mais au lieu de simplement juxtaposer les différents chapitres, j'ai essayé de construire quelques fils conducteurs (comme par exemple le développement de la notion de nombre ou de la démonstration et du système axiomatique) qui guidaient le travail en apparaissant tout au long de l'atelier et en se croisant de temps en temps. Un aspect multiculturel était abordé par un chapitre sur l'ethnomathématique, et l'interdisciplinarité par quelques relations entre les mathématiques, la philosophie et les arts. L'atelier était introduit par une discussion libre sur la nature et l'utilité des mathématiques ; par la suite, nous sommes plusieurs fois revenus sur les questions ayant émergé lors de cette première séance, et nous avons terminé l'atelier par une nouvelle discussion, afin de voir si la conception des élèves sur les mathématiques avait changé suite aux expériences de la semaine.

J'ai essayé de varier les méthodes pédagogiques tout au long de mon atelier. J'ai effectué peu de cours magistraux, j' ai plutôt laissé la place à des discussions libres, à la résolution individuelle de problèmes et aux compétitions en groupes. Je me suis en outre appuyée sur de nombreux supports, depuis la pomme de pin ou l'ananas pour illustrer l'apparition de la série Fibonacci dans la nature, jusqu'aux films et aux logiciels géométriques. Mais une méthode fondamentale et très répandue dans l'enseignement de l'histoire des mathématiques, à savoir la lecture des textes anciens, était totalement absente à cet atelier. Cela s'explique d'une part par le fait qu'à part les Éléments d'Euclide et le Discours de Galilée, il n'en existe pratiquement pas en traduction hongroise, et d'autre part parce que j' ai jugé cet exercice trop difficile : $\mathrm{j}$ 'avais moi-même très peu d'expérience dans la lecture de textes anciens, et je ne disposais alors d'aucune source historique ou didactique qui aurait pu m'orienter dans la conception d'une telle séance.

Quelques éléments de ce premier atelier ont été repris, avec plus ou moins de succès, dans différents contextes : un camp d'été pour collégiens doués en mathématiques, des classes ordinaires de lycéens, un colloque pour enseignants des mathématiques et pour formateurs des enseignants ${ }^{3}$. Dans le même temps, quelques expériences à l'étranger (en France et ailleurs) m'ont permis de découvrir que des recherches existaient en histoire des mathématiques et sur son intégration dans l'enseignement ${ }^{4}$. Ces expériences m'ont incitée à continuer mes réflexions sur le sujet à un niveau plus approfondi.

\section{Réflexions concernant un nouveau cours pour la formation des enseignants}

\subsection{La question des objectifs}

Pour le problème qui nous intéresse ici, à savoir la construction d'un nouveau cours d'histoire des mathématiques pour la formation des enseignants de mathématiques, il est avant tout utile de s'interroger sur ses objectifs. Sont-ils les mêmes que dans le cas d'un atelier pour des élèves du secondaire ? Quels sont la place et le rôle d'un tel cours dans la formation des enseignants ? Quels sont ses rapports à des cours disciplinaires, et à des cours de pédagogie et de didactique ?

Les expériences internationales montrent que les objectifs de l'intégration de l'histoire des mathématiques dans l'enseignement ou dans la formation des enseignants peuvent être très variés.

\footnotetext{
3 Varga Tamás Napok, Budapest, 2011.

${ }^{4}$ Par exemple : Journées ReForEHST, Arras, 2009 ; ESU-6 ( $6^{\text {th }}$ European Summer University ont he History and epistemology in Mathematics Education), Vienne 2010 ; les travaux du groupe M :A.T.H et de la Commission Inter-IREM Épistémologie et Histoire des Mathématiques ; l'étude ICMI History in Mathematics Education.
} 
L'étude ICMI déjà mentionnée traite par exemple à plusieurs reprises des motifs possibles suivants : enrichir la culture générale des élèves/étudiants avec les connaissances sur l'histoire de la discipline ; aider une compréhension meilleure et plus profonde de certains chapitres de mathématiques; transformer la vision sur la nature de la discipline, «humaniser les mathématiques », la présenter comme la production d'une activité humaine ; montrer comment les mathématiques ont pu répondre à divers problèmes au cours de l'histoire ; situer les mathématiques dans une culture plus générale, montrer ses dimensions multiculturelles et ses aspects interdisciplinaires ; améliorer l'attitude des élèves/étudiants par rapport aux mathématiques, etc. La liste est souvent complétée, dans le cas de la formation des enseignants, par la volonté d'enrichir l'arsenal pédagogique des enseignants, et dans certains cas, par leur faire connaître l'histoire de l'enseignement de leur discipline.

Il ressort des nombreuses expériences décrites dans l'étude ICMI, notamment des pays de la « périphérie » par rapport au développement des mathématiques occidentales, un aspect patrimonial de ces cours d'histoire des mathématiques, dont la forme varie en fonction de la culture mathématique locale : un chapitre sur l'école locale de mathématiques (Pologne), l'articulation de la tradition mathématique locale avec la tradition européenne et d'autres grandes traditions (Hong-Kong, Maroc), l'ethnomathématique, en comparant la culture mathématique traditionnelle des peuples locaux aux mathématiques « scolaires» (Brésil, Mozambique).

Cette énumération systématique des objectifs possibles aide à approfondir la réflexion sur le nouveau cours dont la construction est en question. Quels sont alors, parmi ces objectifs, les plus légitimes pour un cours qui s'adresse aux étudiants hongrois en formation pour l'enseignement des mathématiques ?

Faut-il par exemple introduire de nouveaux chapitres mathématiques, comme dans mon premier atelier, dans le cadre d'une formation qui, dans le cas de la Hongrie, assure déjà une éducation disciplinaire assez solide ? Ne faut-il pas plutôt se concentrer sur une meilleure compréhension des chapitres mathématiques déjà connus ? Sur quels thèmes les étudiants en ont-ils besoin ?

Ce cours peut-il aider à développer les compétences didactiques des futurs enseignants, et de quelle manière?

Le travail sur la vision que les futurs enseignants ont de leur discipline semble essentiel pour la formation des enseignants - mais il reste, la plupart du temps, implicite. Un cours d'histoire des mathématiques peut-il contribuer à ce travail ?

Faut-il tenter d'offrir un panorama général de l'histoire de la discipline - dans le cadre d'un cours qui dure environ 30 heures ? Ou bien se limiter à quelques exemples, et profiter de ce temps pour mieux répondre à d'autres objectifs ?

Situer les mathématiques dans la culture générale paraît un objectif pertinent pour la formation des futurs enseignants. Mais quel est le rôle d'une approche multiculturelle ou interdisciplinaire ? En Europe de l'Ouest par exemple, où un des motifs centraux des enseignants à se tourner vers l'histoire des mathématiques est de chercher à aider les élèves issus de familles immigrées, l'aspect multiculturel paraît essentiel. Cette question ne se pose pas au même degré en Hongrie à l'heure actuelle, contrairement à celle de l'interdisciplinarité, étant donné que les enseignants hongrois enseignent presque toujours deux matières.

En général, les questions posées sur les objectifs induisent plusieurs réalisations possibles d'un tel cours d'histoire des mathématiques. Elles permettent aussi de prendre conscience du fait que pour construire un cours efficace et bien intégré dans l'ensemble de la formation, il ne suffit ni de reprendre une expérience réalisée dans un autre contexte et pour un public différent (comme les lycéens volontaires dont il a été question plus haut), ni de copier, sans adaptation au contexte local, les exemples élaborés dans d'autres pays.

Par ailleurs, ces réflexions sur les objectifs débouchent rapidement sur d'autres questions définies par des contraintes de types très différents. L'une d'entre elle et à laquelle j'ai déjà fait allusion, est celle des sources primaires et secondaires, disponibles pour une telle formation. C'est le problème que nous allons maintenant aborder. 


\subsection{Un problème de sources}

En me préparant à mon premier atelier pour lycéens, j'ai examiné la littérature disponible en langue hongroise sur l'histoire des mathématiques. Cette offre est très restreinte, et limite de manière importante les possibilités d'intégration de l'histoire des mathématiques dans l'enseignement.

Comme je l'ai signalé plus haut, le premier problème tient au fait que très peu de sources primaires sont accessibles en langue hongroise. Les traductions des grands textes mathématiques historiques se limitent essentiellement aux Éléments d'Euclide et aux grands dialogues de Galilée ${ }^{5}$. Les textes mathématiques écrits en langue hongroise sont nés en grande majorité à la fin du $19^{\mathrm{e}}$ et au $20^{\mathrm{e}}$ siècle, et ils sont souvent trop difficiles pour les étudiants. Par ailleurs bien des textes de cette époque produits par des mathématiciens hongrois ont été publiés dans les revues internationales, et donc rarement écrits en hongrois.

La situation n'est pas bien meilleure au niveau des sources secondaires : outre les traductions de quelques grands classiques (comme Van der Waerden ou Neugebauer) ${ }^{6}$ et de quelques grandes encyclopédies, en majorité anciennes (anglais, russes ou hongrois) ${ }^{7}$, l'offre se limite en gros aux œuvres d'Árpád Szabó sur les mathématiques grecques ${ }^{8}$, et à quelques textes sur les deux Bolyais ${ }^{9}$. Sur l'histoire des mathématiques hongroises, les œuvres de Barna Szénássy peuvent encore servir comme sources $^{10}$, mais ses recherches s'arrêtent au début du $20^{\mathrm{e}}$ siècle, au moment de l'émergence des recherches mathématiques vraiment significatives en Hongrie. En résumé, les sources disponibles en langue hongroise sont peu nombreuses et en grande majorité anciennes.

La plupart des expériences pédagogiques que j'ai pu observer, et qui ne se limitent pas à un cours magistral mais cherchent à rendre actifs les étudiants, s'appuient en particulier sur la lecture des textes anciens. Les étudiants peuvent aussi être invités à effectuer une recherche individuelle dans la littérature primaire et secondaire sur l'histoire des mathématiques ${ }^{11}$. Mais dans les conditions qui sont les nôtres en Hongrie et que j' ai rapidement décrites ci-dessus, un cours construit sur la lecture régulière d'extraits de sources primaires ou un travail de recherche individuel des élèves ou des étudiants devient très difficile, simplement à cause de l'absence de sources.

Ce problème ne se limite pas aux sources utilisables directement par les élèves et les étudiants, mais concerne aussi celles qui devraient aider l'enseignant ou le formateur à concevoir son cours. En effet, on trouve très peu de documents dans les bibliothèques hongroises, même en langue étrangère, et surtout peu de documents récents. L'accessibilité de plus en plus grande des sources sur le réseau électronique réduit largement ce problème, mais elle pose de nouveaux problèmes, celui de la trop grande diversité des sources et de l'orientation dans cette diversité. En général, l'absence d'une communauté et d'un discours de chercheurs historiens qui aiderait à la production des sources pour la formation et à l'orientation parmi des sources accessibles, demeure une limite significative.

\footnotetext{
5 Euklidész 1983, Galilei 1986.

6 Van der Waerden 1997, Neugebauer 1984.

7 Par ex. Sain 1986, Filep 1997, Ribnyikov 1968, Juskevics 1982, Struik 1958. Un document intéressant pour l'éducation est un recueil des tâches historiques de Lévrádi-Sain 1982.

8 Par ex. Szabó 1978.

${ }^{9}$ Le père, Farkas Bolyai (1775-1856), qui a gardé une amitié avec Gauss depuis leurs études à Göttingen, avait plusieurs résultats mathématiques originaux. Son fils, János Bolyai (1802-1860) est devenu célèbre pour l'invention de la géométrie hyperbolique qu'il a publiée en 1831 (presque en même temps que l'autre inventeur, le russe mathématicien Lobatchevski). Malgré leurs résultats, les Bolyais étaient solitaires et peu reconnus pendant leurs vies, leur œuvre n'était découvert par le monde scientifique qu'à la fin du $19^{\mathrm{e}}$ siècle.

10 Surtout Szénássy 1970.

${ }^{11}$ Un exemple intéressant est le cours développé par Mathieu Husson et Alain Bernard à l'Université de Créteil entre 2011 et 2013, et dont j'ai pu observer quelques séances en 2012-13. Pour des détails sur ce cours voir (Bernard, de Montgolfier, Roux 2015, partie 1).
} 
Pour la construction de mon programme de colonie, j'ai utilisé trois types de sources, à l'époque uniquement en langue hongroise : des grandes encyclopédies chronologiques de l'histoire des mathématiques; quelques sources pour l'enseignement, pas nécessairement historiques, mais avec une possibilité d'adaptation (par ex. sur le nombre d'or, la suite de Fibonacci...) ; et enfin, quelques textes de vulgarisation des mathématiciens hongrois des années 1940-60, tels que les Jeux avec l'infini de Rózsa Péter, les Dialogues sur les mathématiques, ou les Lettres sur la probabilité d'Alfréd Rényi, enfin des textes de philosophie des mathématiques, comme les Preuves et réfutations d'Imre Lakatos), parmi d'autres. Ces derniers textes ne sont pas des œuvres d'histoire des mathématiques, ils donnent néanmoins une certaine approche quasi historique des mathématiques et peuvent ainsi inspirer une approche historique dans l'enseignement.

Cette troisième catégorie de sources est devenue le centre de mes intérêts dans le contexte de mes recherches actuelles, en doctorat, et servira comme base de ma proposition d'un nouveau cours, comme on le verra plus loin.

Il apparaît en effet que les différents problèmes évoqués plus haut, des sources utilisés pour intégrer l'histoire des mathématiques dans l'enseignement, ne sont pas spécifiques à la Hongrie. Dans leur contribution dans ce même volume, Bernard et Husson ont souligné les problèmes liés aux choix des sources disponibles pour l'intégration d'un aspect historique dans l'enseignement des sciences et dans la formation des enseignants. ${ }^{12}$ L'étude ICMI précitée présente une grande diversité d'exemples du monde entier dans le chapitre consacré à la formation des enseignants, et confirme ainsi que le problème des sources est global, même s'il se présente sous des formes variées selon les pays. Au Royaume-Uni par exemple $^{13}$, où comme en France la recherche en histoire des mathématiques repose sur une tradition bien établie, les formateurs se concentrent sur l'orientation des étudiants parmi les diverses sources disponibles : pour la formation présentée dans le livre, on indique qu'une bibliographie contenant plus de 250 livres était offerte aux étudiants (dont $80 \%$ des titres ont été publiés dans les 15 dernières années) et une sélection de sites pertinents était aussi proposée. Dans d'autres pays, par contraste, c'est plutôt l'absence des sources qui pose problème : l'auteur de Hong-Kong ${ }^{14}$ explique par exemple qu'en l'absence de sources primaires, les étudiants basent leur travail sur des sources secondaires de qualité très inégale ; il précise encore que le formateur lui-même, sans formation en histoire des sciences, a une difficulté à les orienter parmi les sources. Comme je l'ai souligné plus haut, l'intégration de l'histoire des mathématiques dans l'enseignement suit souvent des objectifs patrimoniaux - mais la nature des sources utilisées varie bien sûr selon la culture locale des mathématiques. Ainsi, quand le formateur du Maroc $^{15}$ défend le principe de s'appuyer dans tous les cas sur la lecture des textes originaux, l'expérience de Mozambique ${ }^{16}$ profite plutôt de l' " oral history », en référence à des entretiens avec des personnes âgées ou n'ayant pas reçu une éducation formelle.

Apparemment donc, le problème des sources disponibles dans le cas de la Hongrie est similaire aux autres pays de la «périphérie » où la recherche en histoire des mathématiques a peu de tradition. On verra ainsi que la solution que je proposerai plus loin (quatrième partie) se base sur de telles considérations patrimoniales, à partir des textes de vulgarisation hongrois présentés ci-dessus.

Auparavant, j'en reviens au second aspect qui me conduit à privilégier les sources hongroises dont j'ai parlé plus haut et je présente ci-dessous mes recherches actuelles sur et à partir de ces sources. Ces recherches ont en effet émergé en partie de mes expériences liées à mon premier atelier d'histoire des mathématiques, comme on l'a vu, et elles représentent une source d'inspiration essentielle pour la conception du cours envisagé.

\footnotetext{
12 Bernard, Brechenmacher, Husson 2015.

13 David Lingard in Fauvel-Maanen 2000, p. 117-122.

14 Chun-Ip Fung ibid. p. 110-113.

15 Abdellah El Idrissi, ibid. p.124-127.

16 Abdulcarimo Ismael, ibid. p. 122-124.
} 


\section{Recherches sur l'histoire et l'enseignement des mathématiques}

Mon choix de sujet de thèse n'est en effet pas indépendant de mes expériences précédentes d'enseignement de l'histoire des mathématiques, et plus particulièrement des œuvres de vulgarisation et des œuvres philosophiques hongroises, que j'ai mentionnées plus haut comme sources d'inspiration pour mon atelier. Mes recherches ont pris comme point de départ la reconnaissance du fait que ces textes représentaient une culture plus ou moins cohérente, une conception des mathématiques et de son enseignement partagée par leurs auteurs : mathématiciens, enseignants et philosophes hongrois. J'ai identifié ces textes, jusqu'ici peu analysés, comme des documents représentatifs d'une « tradition hongroise » de l'enseignement des mathématiques. Paradoxalement, l'existence de cette tradition est presque devenue en Hongrie un lieu commun et de nombreux mathématiciens, didacticiens, formateurs d'enseignants veulent la préserver et la transmettre, mais sans vraiment pouvoir la caractériser ou la décrire en détail, entraînant ainsi des difficultés dans la transmission.

La reconnaissance de ce fait a d'abord induit chez moi un changement de point de vue sur ces œuvres. Elles étaient à l'origine mes sources secondaires, inspirant la conception de mon atelier d'histoire des mathématiques, et elles sont devenues des objets d'étude, sources primaires d'une analyse historico-didactique.

L'étude historique centrée sur ces auteurs démontre l'existence de liens personnels importants entre eux. Certaines recherches récentes, centrées plus sur la biographie d'Imre Lakatos que sur l'histoire de l'enseignement des mathématiques, ${ }^{17}$ ont déjà attiré l'attention sur un fait important : dans les années 1940, des mathématiciens de premier ordre comme László Kalmár, Rózsa Péter, Alfréd Rényi, et les futurs réformateurs de l'enseignement des mathématiques János Surányi et Tamás Varga ont participé à un cercle d'intellectuels réunis autour du pédagogue-psychologue Sándor Karácsony, discutant des questions liées à l'éducation. Le futur historien des mathématiques Árpád Szabó, et le futur philosophe des sciences Imre Lakatos étaient aussi en relation avec Karácsony, et gardaient un contact avec plusieurs des mathématiciens mentionnés. Même George Pólya, qui ne vivait pas en Hongrie depuis la première guerre mondiale, avait un certain contact, certes plus éloigné, avec ce groupe.

Les mathématiciens du cercle de Karácsony gardaient tous un intérêt pour les questions de l'enseignement des mathématiques, et plus tard, dans les décennies 1960 et 1970, ils ont tous soutenu le mouvement de réforme de Tamás Varga. La réforme mise en place par ce dernier et ses collègues en 1978 au niveau de l'école primaire et du collège a profondément transformé l'éducation des mathématiques en Hongrie, et son influence reste présente jusqu'à nos jours.

Mon travail de thèse est centré sur l'étude de ce mouvement de réforme de Varga. En comparant cette réforme aux réformes françaises qui leur sont contemporaines (1969-70, la réforme des « mathématiques modernes » en 1977), je cherche à identifier d'une part leurs caractéristiques communes, issues des discours internationaux de l'époque ${ }^{18}$ et d'autre part les différences entre les deux pays. J'essaye de montrer que les spécificités de la réforme hongroise remontent aux traditions d'enseignement des mathématiques plus anciennes, représentées entre autres par le groupe des mathématiciens participant au cercle de Karácsony dont il a été question plus haut.

Une analyse textuelle des divers écrits de ces auteurs permet d'identifier quelques principes importants de leur conception des mathématiques et de leur enseignement. En comparant ces principes aux caractéristiques des programmes et des manuels scolaires de Varga (des années 1970), une continuité profonde peut être dévoilée dans cette tradition hongroise.

\footnotetext{
17 Gurka 2001, Máté 2006.

18 Par ex. la volonté de suivre les changements des mathématiques contemporaines dans l'enseignement; l'influence de Piaget ou de Dienes.
} 
Une grande partie de ces textes vise la vulgarisation des mathématiques. Dans Jeux avec l'infini par exemple, Rózsa Péter s'adresse à des lecteurs non mathématiciens, avant tout aux littéraires ; elle invite les lecteurs à un long voyage de découverte des mathématiques, des notions les plus simples jusqu' aux résultats modernes à son époque, comme les théorèmes de Gödel et de Church. Elle écrit dans un style quasi littéraire, utilise très peu de formules ou de démonstrations formelles et se concentre sur les processus de découverte des mathématiques, sur une dialectique des problèmes, des questions et des tentatives de réponse. Les œuvres de vulgarisation de Rényi prennent souvent la forme de pastiche : dans les Dialogues sur les mathématiques par exemple, il évoque la nature des mathématiques sous forme de dialogue platonicien ou galiléen, dans les Lettres sur la probabilité, il écrit des lettres fictives au nom de Pascal, adressées à Fermat, pour présenter les bases de la théorie des probabilités. László Kalmár, quant à lui, n'a pas publié d'œuvre de vulgarisation à proprement parler - mais plusieurs de ses lettres, expliquant des questions mathématiques difficiles à ses collègues ou à ses amis non-mathématiciens sont devenues célèbres à cause de leur style intuitif, original, et leur grande force explicative. ${ }^{19} \mathrm{Il}$ parle en outre, dans plusieurs de ses conférences, de la nature des mathématiques, souvent en lien avec les questions d'éducation. Certaines recherches récentes ${ }^{20}$ montrent que ces conférences pouvaient avoir une très grande influence, pas seulement sur des mathématiciens et des enseignants mais aussi sur le jeune Imre Lakatos, futur philosophe des mathématiques et des sciences. L'œuvre peut-être la plus connue de Lakatos, Preuves et réfutations prend la forme d'un dialogue fictif d'une classe de mathématiques, et présente un processus dialectique, où les notions, les définitions, les théorèmes et les démonstrations progressent parallèlement, en s'appuyant sur de nombreux exemples et contre-exemples. Mentionnons enfin les œuvres de George Pólya qui, au lieu de la nature déductive des mathématiques, met l'accent sur l'importance des processus d'invention et de résolution de problèmes, et propose des outils d'apprentissage pour devenir plus efficace dans ces processus.

Au total, j'ai établi à partir d'une lecture comparative que plusieurs principes communs ressortent de ces textes, malgré leur diversité apparente :

1. Les mathématiques ne sont pas considérées comme statiques et éternelles, mais plutôt comme une création de l'esprit humain, quelque chose qui change et évolue sans cesse. Les élèves doivent également être accompagnés selon le même processus évolutif de création.

2. La source des mathématiques est l'intuition et l'expérience (non limitée aux observations physiques réelles). Il est important de développer l'intuition à l'aide de nombreuses expériences riches et diverses à tous les niveaux de l'éducation.

3. L'activité mathématique est essentiellement dialogique, il s'agit d'une série de questions, de problèmes et de tentatives pour y répondre. L'enseignement des mathématiques est une activité conjointe de l'élève et de l'enseignant.

4. Tout formalisme inutile est découragé, l'utilisation d'un langage formel ne doit être introduite qu'après une préparation appropriée.

5. Le but de l'enseignement des mathématiques n'est pas de transmettre de manière irréfléchie des recettes de calculs mais de fournir une initiation au processus de la création mathématique, et par conséquent d'éduquer les gens à réfléchir.

6. Le processus de la création mathématique est en relation étroite avec le jeu, et dans cet aspect ludique, c'est la nature artistique des mathématiques qui se manifeste. ${ }^{21}$

Mon travail porte maintenant sur l'analyse didactique des documents et principalement des programmes et des manuels scolaires de la réforme de Varga : j'essaye de montrer comment la tradition hongroise de conception des mathématiques et de son enseignement, présentée ci-dessus, influence la construction

\footnotetext{
19 Certaines de ces lettres ont été publiées après sa mort dans Kalmár 1986.

20 Gurka 2001, Máté 2006.

${ }^{21}$ Cette analyse était présentée en détails au premier colloque sur Mathematical Cultures (Londres, septembre 2012 , https://sites.google.com/site/mathematicalcultures/conference-1).
} 
des sources didactiques de l'équipe de Varga. De surcroît, cette recherche et cette articulation des approches didactiques et historiques sont susceptibles d'inspirer une nouvelle conception de cours pour la formation des enseignants, dont je présente l'ébauche au point suivant.

\section{Une conception possible d'un cours?}

L'analyse précédente montre que les textes de vulgarisation dont il a été question présentent une conception particulière et assez cohérente des mathématiques et de son enseignement. Compte tenu des difficultés cernées plus haut (partie II), une idée s'impose alors naturellement : l'étude de ces textes dans le cadre de la formation des enseignants serait probablement susceptible de contribuer à former la pensée des futurs enseignants sur ces questions. Cette conception humaniste et évolutive semble d'ailleurs assez proche de celle que l'on retrouve dans les expériences intégrant l'histoire des mathématiques.

En même temps, ces textes peuvent être aussi vus comme des documents historiques qui témoignent d'une culture particulière propre à une tradition hongroise d'enseignement des mathématiques.

Ce double point de vue motive l'idée de s'appuyer sur les textes évoqués précédemment pour concevoir un cours dans la formation des enseignants des mathématiques. Un cours basé sur leur lecture aurait beaucoup d'avantages. Avant tout, ils sont facilement disponibles et lisibles pour les étudiants, pas seulement parce qu'ils sont écrits en hongrois, mais aussi parce qu'il s'agit de textes relativement modernes dont la majorité étaient, dès l'origine, destinés à être des œuvres vulgarisatrices, adressées donc au grand public. Il s'agit donc d'un corpus qui permet de contourner la majorité des problèmes mentionnés plus haut en lien à l'utilisation des sources historiques. Deuxièmement, ces textes offrent de très bonnes occasions de travailler sur la conception que les étudiants ont des mathématiques, et cela pour la majorité des œuvres, en lien direct avec des questions d'éducation. Enfin, en les traitant comme des documents historiques, ils peuvent aider à prendre conscience d'une tradition d'enseignement des mathématiques importante en Hongrie. Par cet aspect patrimonial, la place d'un tel cours, basé sur l'étude des œuvres de vulgarisation (ou philosophiques) des mathématiciens hongrois, pourrait être facilement définie dans le contexte de la formation des enseignants en aidant justement à la transmission - assez problématique, comme on l'a vu - de la tradition hongroise de l'enseignement des mathématiques.

Un tel cours aurait donc beaucoup d'intérêt - en même temps, on ne peut pas négliger les limites de cette conception. Dans son état actuel, elle n'est pas apte à répondre à nombreux objectifs qui peuvent être raisonnablement visés par rapport à un cours d'histoire des mathématiques. Par exemple, un cours centré sur des textes hongrois du milieu du $20^{\mathrm{e}}$ siècle ne suffit évidemment pas pour transmettre une culture générale en histoire des mathématiques, ni pour présenter un aspect multiculturel des mathématiques (Il peut donner cependant lieu à des discussions sur les questions d'interdisciplinarité, surtout entre arts, lettres et mathématiques, sujet particulièrement cher à R. Péter et à T. Varga). Un tel cours ne permet pas non plus de traiter des difficultés de lecture et d'interprétation des textes mathématiques plus anciens ou moins accessibles, et ne facilite pas l'initiation des étudiants à la recherche autonome, en raison du nombre assez limité de textes.

Cette approche doit donc encore être développée, pour pouvoir être intégrée dans un cours complet et cohérent. Cependant, quelques expériences ponctuelles récentes m'ont confirmé l'intérêt de travailler sur ces textes dans les cadres de la formation initiale ou continue des enseignants.

Une première expérience a été réalisée dans le cadre d'une formation continue adressée aux enseignants français (de mathématiques, de lettres et d'histoire) de la région parisienne, en novembre 2012 et en janvier 2014. Cette formation, organisée par Alain Bernard, est liée au projet de recherche en histoire des sciences intitulé «Séries de problèmes au carrefour des cultures ». ${ }^{22}$ Dans le cadre

\footnotetext{
22 Mené au sein du Labex HASTEC. Voir le carnet scientifique du projet : http://problemata.hypotheses.org/172, http://problemata.hypotheses.org/225.
} 
de ce projet, des historiens des sciences travaillant sur différentes époques et cultures étudient les textes qu'on appelle "séries de problèmes »: il s'agit en général de sources où on peut identifier un recueil de problèmes ou de questions-réponses, et où leur succession et leur mise en ordre semble jouer un rôle important dans la construction du texte. Chaque demi-journée constitutive des trois jours de formation était animée par différents membres du groupe, présentant un exemple de texte et son contexte historique.

Ma première analyse dans ce contexte a porté sur deux chapitres du livre Jeux avec l'infini de Rózsa Péter. L'extrait choisi décrit une situation d'enseignement dans une classe de $6^{\mathrm{e}}$, issue de l'expérience personnelle de l'auteur. La notion de « série de problèmes » a permis de dévoiler une structure complexe et sophistiquée derrière ce texte, en apparence simple et transparent. La présentation du contexte historique, et la comparaison avec d'autres exemples présentés pendant la formation ont permis d'illustrer la complexité d'une tradition d'enseignement des mathématiques. Simultanément, la situation d'enseignement décrite dans le texte analysé a permis aux enseignants d'articuler l'analyse du texte avec leur propre expérience professionnelle.

La conception de cette formation a été développée progressivement ${ }^{23}$; en outre, après les expériences des deux premières sessions, nous envisageons avec Alain Bernard de compléter la formation par un groupe de travail qui permettrait aux enseignants participants d'approfondir leurs connaissances sur les séries de problèmes et de développer des réflexions sur leur pratique enseignante ${ }^{24}$.

L'analyse de l'extrait de Jeux avec l'infini a été reprise dans un contexte tout à fait différent, lors d'une séance proposée à Bath, aux étudiants anglais de la formation initiale des enseignants de mathématiques, sur l'invitation de Snezana Lawrence. Il s'agissait d'un cours centré sur les questions didactiques plus que d'histoire des mathématiques, où j'étais invitée à m'exprimer sur « la tradition de la résolution des problèmes dans l'enseignement hongrois des mathématiques ». Dans la première partie de cette séance, après une courte introduction du contexte historique, j'ai présenté l'analyse de la démarche de Rózsa Péter, en la mettant en parallèle avec How to solve it ? de G. Pólya, qui reste un auteur très connu dans le monde anglo-saxon. Dans une deuxième partie, j'ai proposé de résoudre quelques problèmes issus de la pratique contemporaine de quelques enseignants hongrois, et qui pouvaient être perçus comme des continuations possibles de la série de Rózsa Péter. Cette construction de la séance a permis d'illustrer la richesse de cette tradition et d'expérimenter certains de ses éléments en pratique, faisant ainsi réfléchir les étudiants sur des questions didactiques.

Ces deux expériences - bien que ponctuelles - sont de bon augure pour les perspectives d'un cours basé sur les textes des mathématiciens hongrois. L'idée d'un tel cours émane des réflexions menées autour de la construction d'un cours d'histoire des mathématiques. Néanmoins, l'expérience de Bath montre que même en étudiant ces textes dans le cadre d'un cours de didactique, sans envisager de l'intégrer dans un cours complet axé sur l'histoire, il peut utilement approfondir les réflexions des étudiants sur l'enseignement.

\section{Références}

Bernard A., Brechenmacher F., Husson M. (2015) « Points cardinaux pour la conception de formations universitaires pluridisciplinaires en épistémologie et histoire des sciences pour les enseignants du secondaire, ou comment s'appuyer sur des dilemmes. » Dans ce volume, article 05004.

Bernard A., de Montgolfier S., Roux-Goupille, C. (2015), « Développer localement une pratique collaborative centrée sur les enseignements SHS pour des étudiants en sciences et techniques. » Dans ce volume, article 02004.

\footnotetext{
23 Voir les billets d'annonce des deux sessions 2012-13 (http://problemata.hypotheses.org/172) et 2013-14 (http: //problemata.hypotheses.org/225).

${ }^{24}$ Le principe de ce groupe de travail est résumé ici : http://problemata.hypotheses.org/268 Nous avons présentés le projet à l'école d'été ESU-7 (Coppenhague, le 15 juillet 2014, http: //conferences . au .dk/ESU-7/).
} 
Euklidész (1983) Elemek. (Mayer Gy. trad.) Budapest : Gondolat.

Fauvel J., van Maanen J (2000) History in Mathematics Education. New ICMI Study Series 6. Dordecht/Boston/London : Kluwer Academic Publishers.

Galilei G. (1986) Matematikai érvelések és bizonyítások. (Dávid G. trad.) Budapest : Európa Kiadó.

Gurka D. (2001) Kalmár László szerepe Lakatos Imre matematikafilozófiájának alakulásában. Recepció és kreativitás http://www.phil-inst.hu/recepcio/htm/3/310_belso.htm

Juskevics (Juskevic) A. P. (1982), A középkori matematika története. (Nagy I., Wirth L. trad. de l'édition russe de 1961 : История математики в средние века $=$ «Histoire des mathématiques au Moyen Âge ». Moscou, Budapest : Gondolat.

Kalmár L. (1986) Integrállevél. Budapest : Gondolat.

Lakatos I. (1984) Preuves et Réfutations : essai sur la logique de la découverte mathématique. Paris : Hermann, 1984. (Ouvrage original publié en 1963 sous le titre Proofs and refutations. London : Nelson.)

Máté A. (2006) Árpád Szabó and Imre Lakatos, Or the relation between history and philosophy of mathematics. Perspectives on Science 14.3 282-301.

Freud R. ed (1981) Nagy pillanatok a matematika történetében. Budapest : Gondolat.

Neugebauer O. (1984) Egzakt tudományok az ókorban. Budapest : Gondolat. (Guman I. trad. de la deuxième édition anglaise de 1957 : The exact sciences in antiquity Providence, RI : Brown University Press.)

Péter R. (1977) Jeux avec l'infini (Kassai G. trad.). Paris : Éditions du Seuil. (Ouvrage original publié en 1944 sous le titre Játék a végtelennel. Budapest : Dante Könyvkiadó).

Pólya G. (1965) Comment poser et résoudre un problème (C. Mesnage trad.). Paris : Dunod. (Ouvrage original publié en 1945 sous le titre How to solve it. Princeton : Princeton University Press).

Rényi A. (2005) Ars Mathematica. Rényi Alfréd összegyújtött írásai. Budapest : Typotex.

Ribnyikov (Rybnikov) (1968) A matematika története. (Oláh Gy. trad. de l'édition russe de 1960 : История математики = «Histoire des mathématiques » Moscou : Izd. Moskovskogo univ.) Budapest : Tankönyvkiadó.

Sain M. (1986) Nincs királyi út! Budapest : Gondolat.

Struik D. (1958) A matematika rövid története. (Auer K. trad. de la deuxième édition anglaise de 1948 : A Concise History of Mathematics. New York : Dover Publications.) Budapest : Gondolat.

Lévrádi L., Sain M. (1982) Matematikatörténeti feladatok. Budapest : Tankönyvkiadó.

Simonyi K. (1981) A fizika kultúrtörténete. Budapest : Gondolat.

Szabó Á. (1978) A görög matematika kibontakozása. Budapest : Magvető.

Szénássy B. (1970) A magyarországi matematika története (a legrégibb idóktól a 20. század elejéig) Budapest : Akadémiai Kiadó.

Waerden B. L. van der (1977) Egy tudomány ébredése : Egyiptomi, babiloni és görög matematika. (Pollák Gy. trad. de l'édition anglaise de 1954 :Science awakening Groningen : P. Noordhoff.) Budapest : Gondolat.

Le carnet scientifique du projet « Séries de problèmes au carrefour des cultures » : problemata.hypotheses.org 